\section{Association between body mass index and health outcomes among Brazilians with and without cardiometabolic comorbidities}

Objective

- To assess the relationship between body mass index (BMI) and its association with self-reported physical function, economic burden and glycaemic status among Brazilian adults

\section{Background}

- In Brazil, the age-standardized prevalence of obesity nearly doubled between 1980 and 2008, ${ }^{1}$ and the relationship between obesity and health outcomes has not yet been adequately studied.

\section{Database}

- The Brazil National Health and Wellness Survey (NHWS) is an annual, Internet-based general health questionnaire administered to a nationwide random stratified sample of adults ( $\geq 18$ years old).

- All respondents provided informed consent prior to participating and the survey was approved by an institutional review board.

- All respondents with non-missing weight data and BM $\geq 18.5 \mathrm{~kg} / \mathrm{m}^{2}(\mathrm{n}=34.252)$ were included. Mean (SD) age was 41 years (15.4) with $51 \%$ being female.

\section{Measurements}

- BMI was calculated using self-reported height and weight. Respondents were categorised into: normal weight (BM $\geq 18.5$ to $<25, n=15,893$ ), overweight ( $B M I \geq 25$ to $<30$, $n=11,960)$, class I obesity (BMI $\geq 30$ to $<35, n=4,423)$, class II obesity (BMI $\geq 35$ to $<40, n=1,269$ ), and class III obesity (BMI $\geq 40 \mathrm{~kg} / \mathrm{m}^{2} \mathrm{n}=707$ ).

- The Diabetes Screening Score was used to categorize respondents into type 2 diabetes (T2D), pre-diabetes, or euglycaemia groups.

- Covariates included sociodemographic, health behaviour and comorbidity measures.

- Self-reported physical function (Physical Component Summary; P(S) was based on responses to the SF-12v2/ SF-36 2,

- Healthcare annual resource use was calculated based on self-reported physician visits or hospitalizations multiplied by the cost from:

- Classificação Brasileira Hierarquizada de Procedimentos Médicos (CBHPM) (Brazilian Hierarchical Classification of Medical Procedures) - private physician costs

Sistema de Gerenciamento da Tabela de Procedimentos, Medicamentos e OPM do SUS (SIGTAP) - public physician costs

União Nacional das Instituições de Autogestão em Saúde (Unidas) - private hospitalization costs

Autorização de Internação Hospitalar do Ministério da Saúde. Secretaria de Atenção à Saúde (SAS): Sistema de Informações Hospitalares no SUS (SIH/SUS; AlH) - public hospitalization costs.

- Indirect costs were calculated by combining absenteeism (number of hours missed in the last week) and presenteeism (number of hours missed in the last week because of health impairment) from the Work Productivity and Activity Impairment questionnaire ${ }^{4}$ multiplied by average annual salaries from the Organisation for Economic Co-operation and Development.

\section{Analyses}

- Descriptive and bivariate analysis: outcomes reported as means and standard deviations. One-way ANOVAs were used to determine whether outcome variables differed across BMI.

- Multivariable analyses: generalized linear models were used to model the association between BMI and outcomes, controlling for covariates. Least-squares means were also reported

- The 'normal weight' group and 'euglycaemia group' were the reference group for the BMl and glycaemic status analyses, respectively.

Results

- In the descriptive and bivariate analysis it was observed that, as BMI increased, PCS decreased, and all costs increased (all $p<0.001$ ).
Multivariable analysis:

PCS (Figure 1): high BMI was negatively associated with physical function. Adjusted means for the euglycaemia, pre-diabetes and T2D groups were all significant $(p<0.001)$

Indirect costs (Figure 2): higher BMI was positively associated with indirect costs. The indirect costs for the pre-diabetes group were lower than for the euglycaemia group, and costs for the T2D group were lower than for pre-diabetes obesity class III and euglycaemia obesity class II.

Physician visit costs (Figure 3): BMl was positively associated with physician costs. Adjusted means for the euglycaemia, prediabetes and T2D groups were all significant $(p<0.001)$

Hospitalization costs (Figure 4): BMI was positively associated with hospitalization costs with the exception of class I obesity, which reported a slight decrease. For the prediabetes group, there was a sharp increase in costs between obese class II and class III. With the exception of class III obesity, costs were higher in the T2D group than the prediabetes and euglycaemia groups.

Figure 1 Adjusted means for the Physical Component Summary across BMI groups and glycaemic status.
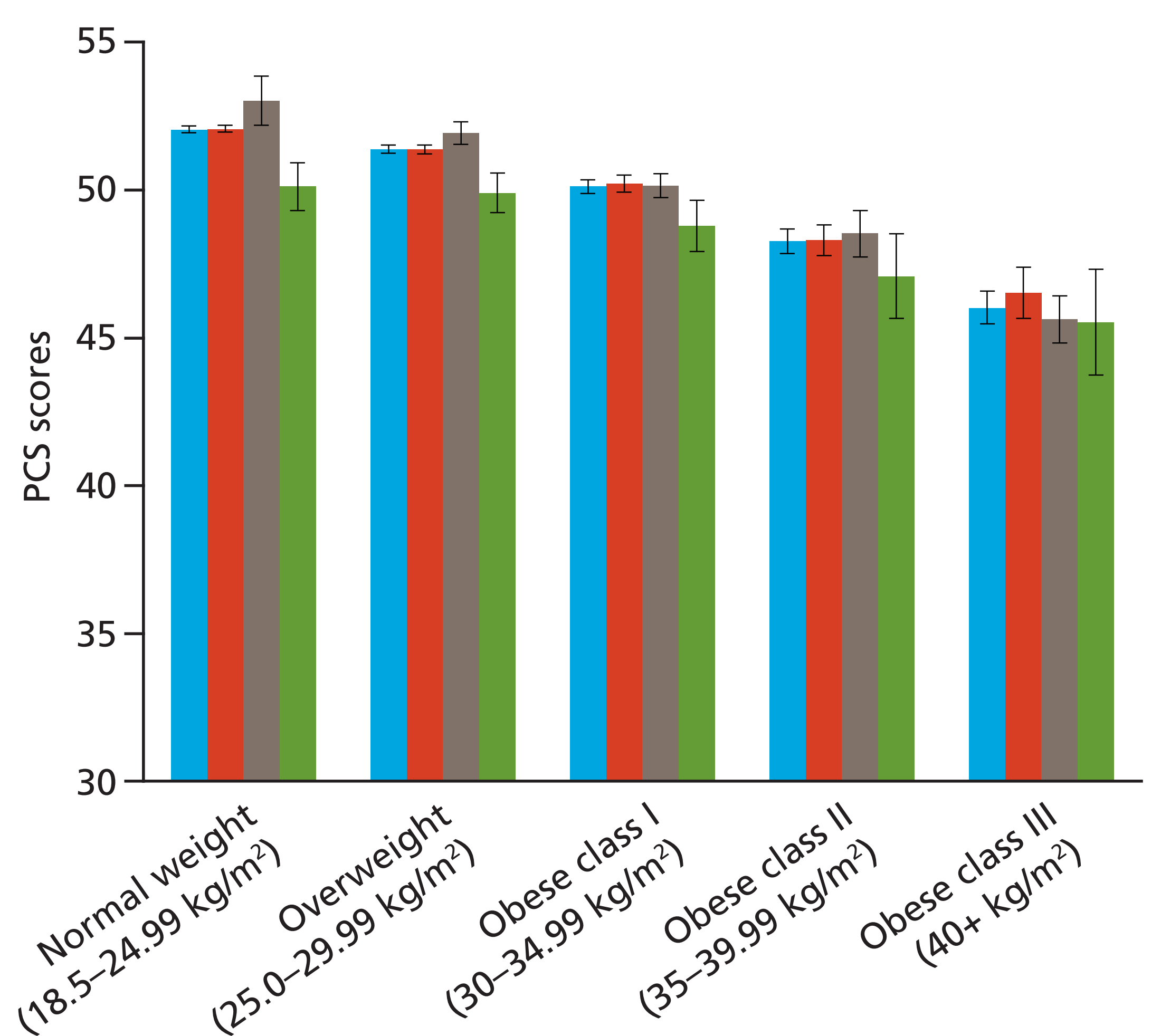

BMI Euglycaemia $\square$ Pre-diabetes Type 2 diabetes Note. Means $(95 \%$ CI) are estimated from generalised linear models (GLMs) with
BMI group as a factor and controlled for age, sex, education exercise, alcohol

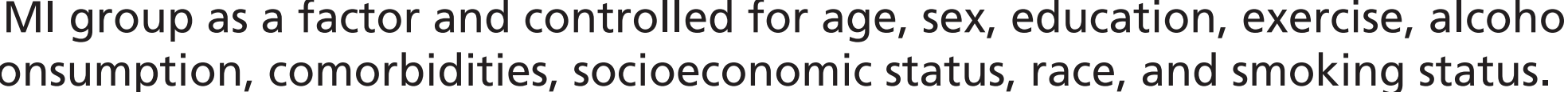
consumption, comorbidilies, socioeconomic status, race, and

Figure 2 Adjusted means for annual indirect costs across BM classes and glycaemic status.

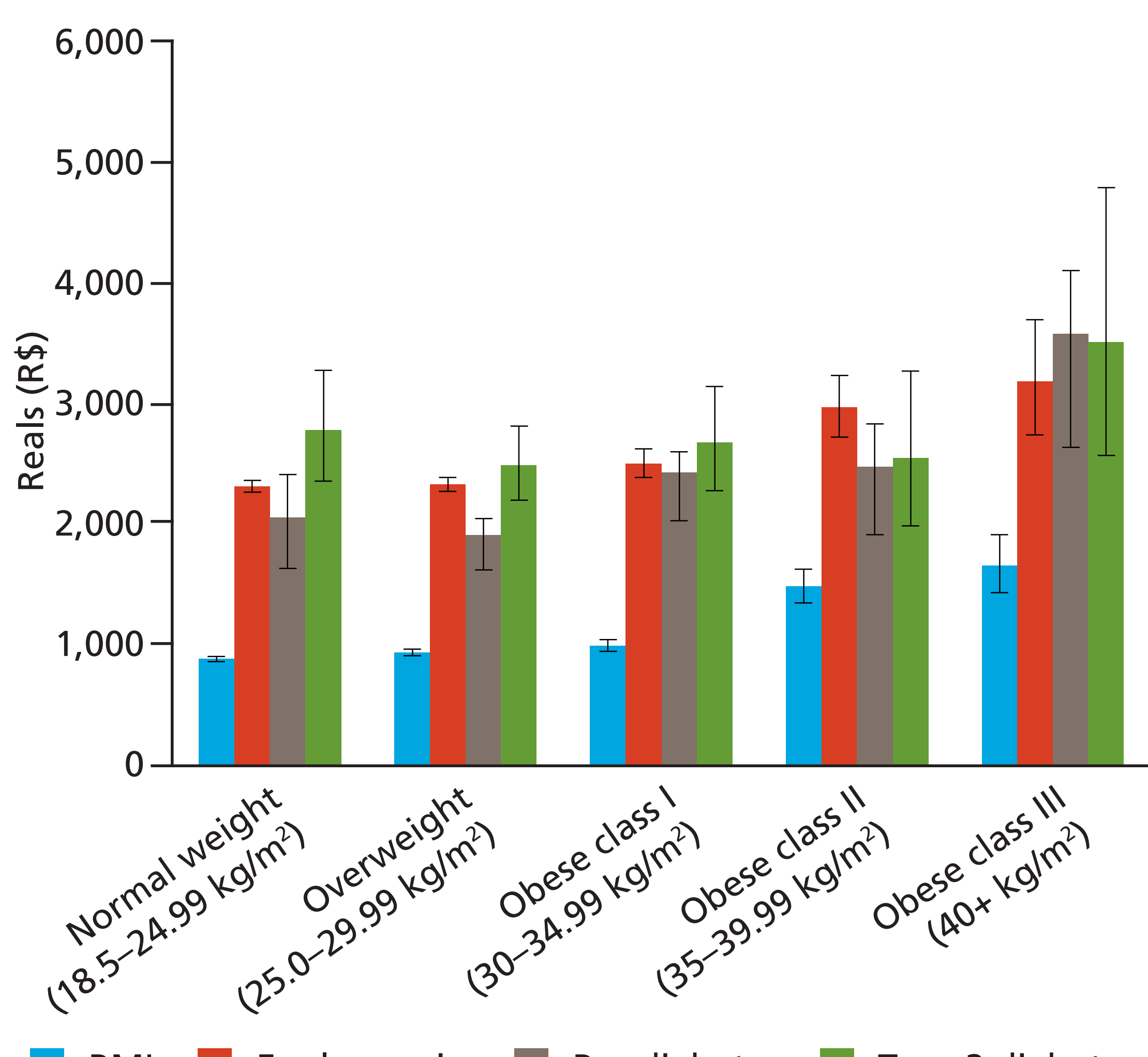

BMI Euglycaemia $\square$ Pre-diabetes Type 2 diabetes Note. Means $(95 \%$ (C) are estimated from generalised linear models (GLMs) with consumption, comorbidities, socioeconomic status, race, and smoking status. BMI, body mass index.
1. Thiago de Menezes Gonçalves são Paulo, Brazil ão Paulo, Brazi

2. Jefferson Siqueira Alves Novo Nordisk Farmaceutica, São Paulo, Brazil

. Ian Kudel Kantar Health, New York, NY, USA

4. Marco DiBonaventura Kantar Health, New York, NY, USA
5. Emil Nortoft Novo Nordisk A/S, Copenhagen, Denmark

6. Brandon Goode São Paulo, Brazil Novo Nordisk A/S, Copenhagen, Denmark

Presenter: Rasmus Skovgaard Novo Nordisk A/S
7. Kristjan Kull

Figure 3 Adjusted means for annual provider costs across BMI classes and glycaemic status.
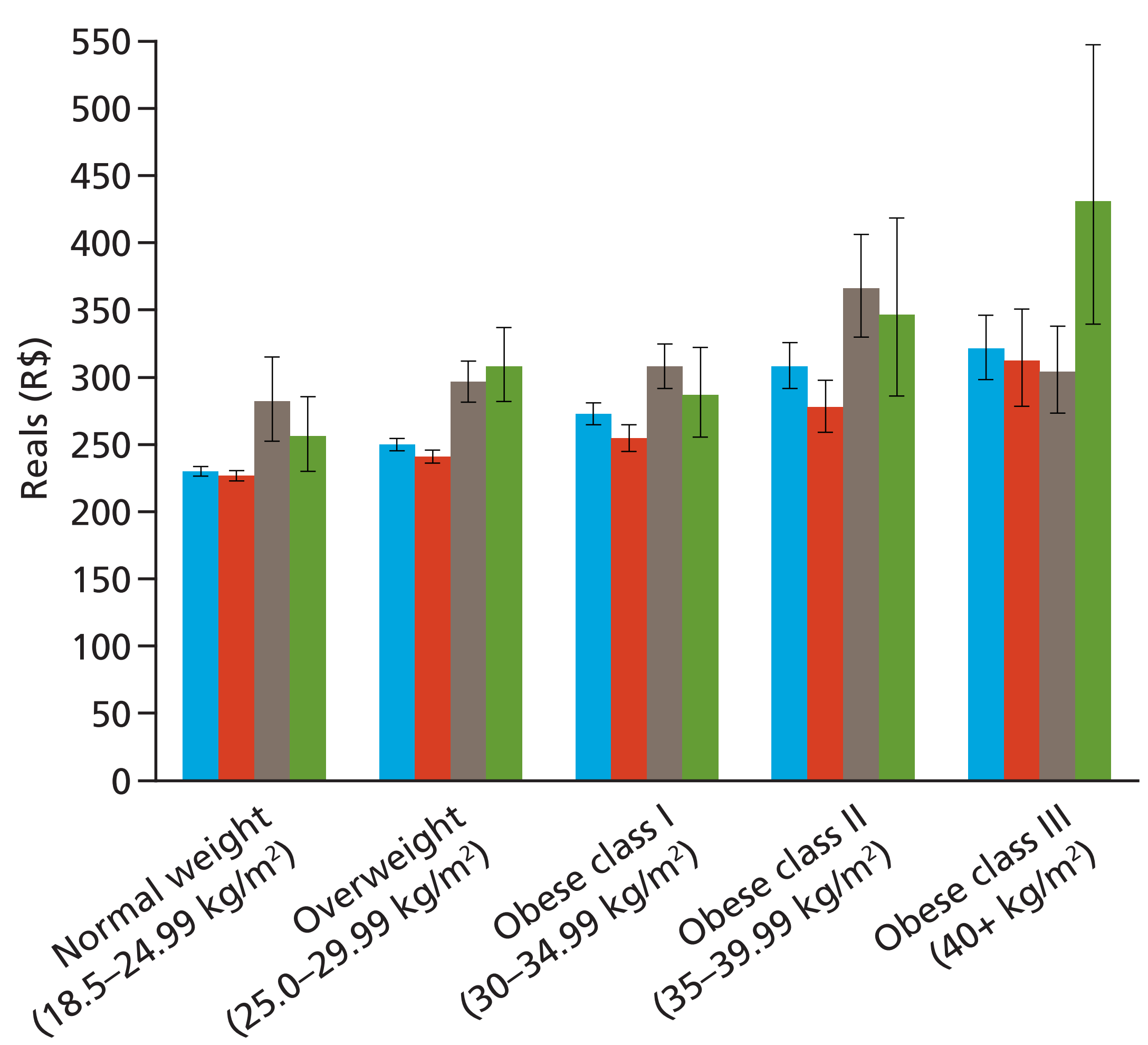

BMI Euglycaemia $\square$ Pre-diabetes $\square$ Type 2 diabetes Note. Means $(95 \%$ (I) are estimated from generalised linear models (GLMS) With BMI group as a factor and controlled for age, sex, education, exercise, alcohol BMI, body mass index.

Figure 4 Adjusted means for annual hospital care across BM classes and glycaemic status.

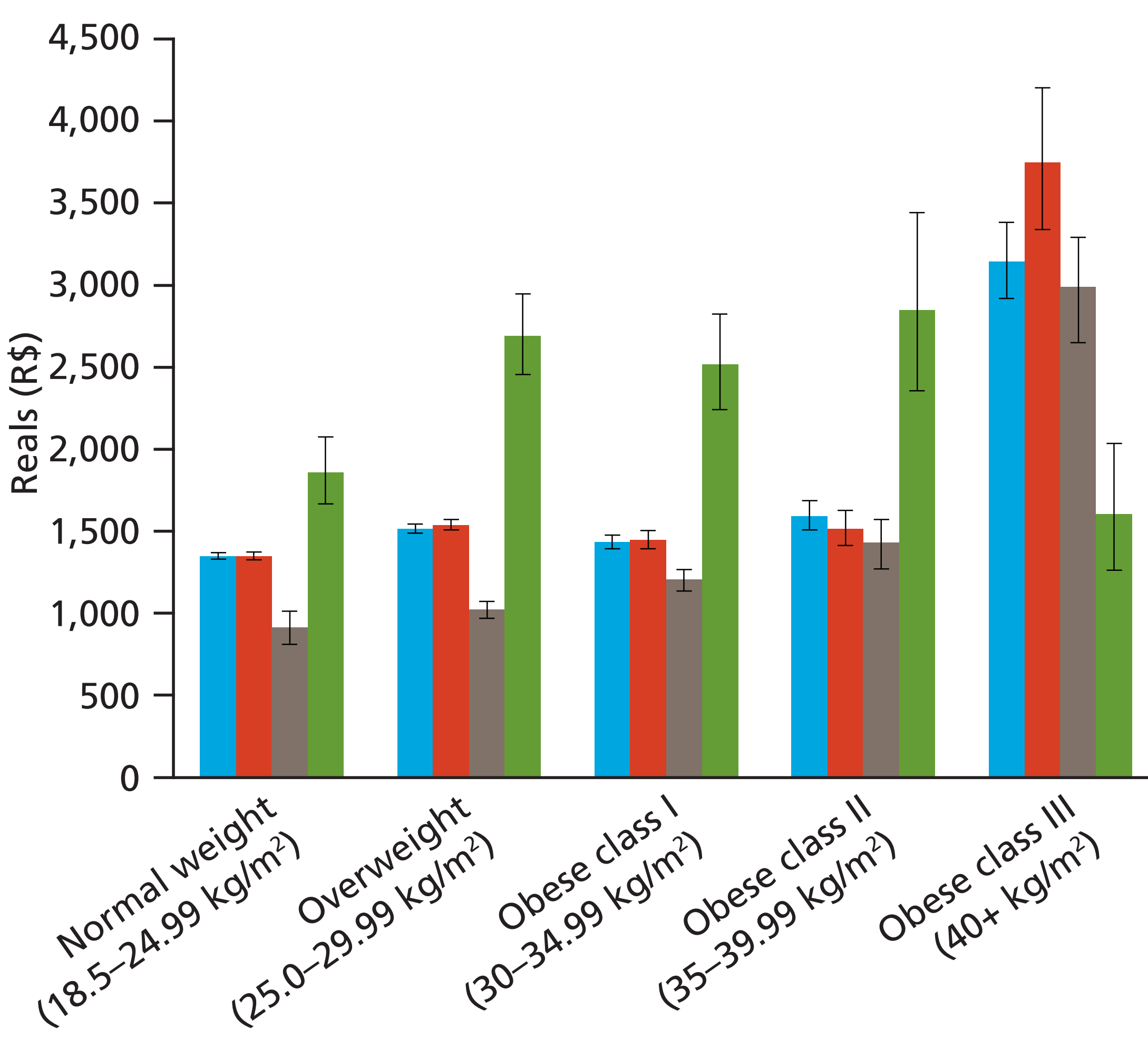

BMI Euglycaemia Pre-diabetes Type 2 diabetes Note. Means $(95 \% \mathrm{Cl})$ are estimated from generalised linear models (GLMs) with BMI group as a factor and controlled for age, sex, education, exercise, alcoho BMI, body mass index.

\section{Limitations}

- All data were self-reported retrospectively.

- The generalizability of our sample to specific populations needs further investigation.

References

Whe Metric Inc : 2000

Rl: Qual Inc 2002:

Reilly et al. Pharmacoeconomics 1993:4:353-65.

\section{Conclusions}

In this sample half of the adults had overweight or obesity. Increasing BMI was negatively associated with PCS and positively associated with costs.

- For glycaemic status there was more variability but generally BMI was negatively associated with PCS and positively associated with costs. These findings indicate that Brazilians with obesity are experiencing associated deleterious obesity are experiencing associated deleterious effects with increased costs. 